\title{
Influence of Stress-strain on the Microstructural Change in the Metallic Glass and Metallic Glass Matrix Composite
}

\author{
*Correspondence to: \\ Lee $\mathrm{MH}$, \\ Tel: +82-32-850-0424 \\ Fax: +82-32-850-0304 \\ E-mail: mhlee1@kitech.re.kr \\ Received May 30, 2015 \\ Revised June 18, 2015 \\ Accepted June 18, 2015
}

\author{
Song-Yi Kim ${ }^{1}$, A-Young Lee ${ }^{1}$, Hye-Ryung $\mathrm{Oh}^{1,2}$, Min-Ha Lee ${ }^{1, *}$ \\ ${ }^{1}$ Rare Metals R\&D Group, Korea Institute of Industrial Technology, Incheon 406-840, \\ ${ }^{2}$ Department Materials Engineering, Hanyang University, Ansan 426-791, Korea
}

\begin{abstract}
At room temperature, metallic glasses deform inhomogeneously by strain localization into narrow bands as a result of yielding due to an external force. When shear bands are generated during deformation, often nanocrystals form at the shear bands. Experimental results on the deformation of bulk metallic glass in the current study suggest that the occurrence of nanocrystallization at a shear band implies the loading condition that induces deformation is more triaxial in nature than uniaxial. Under a compressive stress state, the geometrical constraint strain imposed by the stress triaxiality plays a crucial role in the deformation-induced nanocrystallization at the shear bands.
\end{abstract}

Key Words: Metallic glass, Nanocrystalline, Crystallization, Shear band, Stress triaxiality

\section{INTRODUCTION}

When metallic materials are deformed under a compressive stress state at sub-ambient temperatures and/or high strain rates, grain refinement associated with a phase transformation such as recrystallization is often observed (Ye \& Lu, 1999; Jiang et al., 2003; Lee et al., 2008; Lee et al., 2009; Lee et al., 2014). Metallic glasses (MGs) have received a lot of attention because of their potential applications as high strength materials; however, under uniaxial compressive loading the plastic flow is highly-localized in shear bands, leading to catastrophic failure along one dominant maximum shear plane without global plasticity (Spaepen, 1977; Agron, 1979; Greer, 1995; Liu et al., 1998; Lee et al., 2008; Pauly et al., 2009). The possible deformation mechanisms of bulk metallic glasses (BMGs) have received appreciable attention because of their potential applications as high strength materials (Donovan, 1989; Greer, 1995). In microstructurally isotropic and chemically homogeneous BMGs plastic flow is inhomogeneous at temperatures below the glass transition temperature $T_{g}$, and tends to be localized within a small number of shear bands.
After the imposed shear stress exceeds the shear limit of the BMG, plastic flow occurs in the direction of the maximum shear stress. Several reports clearly show that shear localization occurs by forming shear bands immediately upon the onset of yielding (Conner et al., 2003; Zhang et al., 2003; Lee et al., 2009). Under a uniaxial compressive loading condition, when plastic deformation occurs, MGs usually fail by the formation of highly localized plastic flow in shear bands, leading to catastrophic failure along one dominant maximum shear plane without global plasticity (Spaepen, 1977; Agron, 1979). Several reports show that shear localization occurs by forming shear bands immediately upon the onset of plastic deformation (Conner et al., 2003; Zhang et al., 2003) and the results of atomistic simulations of deformation in MGs also show the important influence of multi-axial stress states on yielding (Lewandowski \& Lowhaphandu, 2002; Lee, 2003; Lund \& Schuh, 2003). As the shear bands propagate during deformation, the atomic mobility inside the shear bands is enhanced and the possibility of structural changes increases within the shear bands (Deng et al., 1989). Interestingly, deformation-induced crystallization of amorphous phase

This work was supported by the Industrial Technology Innovation Program funded by the Ministry of Trade, Industry and Energy (MOTIE) and Korea Institute of Technology Evaluation and Planning (KETEP).

( ) This is an open-access article distributed under the terms of the Creative Commons Attribution Non-Commercial License (http://creativecommons.org/licenses/by-nc/4.0) which permits unrestricted noncommercial use, distribution, and reproduction in any medium, provided the original work is properly cited.

Copyrights (c) 2015 by Korean Society of Microscopy 


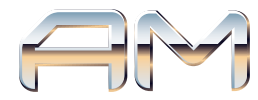

under hydrostatic pressure has been experimentally observed in Zr- and Al-based MGs (Ye \& Lu, 1999; He et al., 2002) and numerically postulated in simple amorphous systems (Lee et al., 2004; Boucharat et al., 2005). Furthermore, the formation of nanocrystals in Al-based amorphous ribbons plastically deformed by bending and Zr-based metallic glass alloys subject to nanoindentation has been reported (Kim et al., 2002; Jiang et al., 2003; Lee et al., 2008; Lee et al., 2009). Some experimental evidences for deformation-induced nanocrystallization have been reported previously based on transmission electron microscopy (TEM) results under locally constraint deformation conditions such as bending (Chen et al., 1994), rolling (Jiang et al., 2003) or nano-indentation (Vaidyanathan et al., 2001; Kim et al., 2002).

In spite of the previous observations, the structure of the shear bands and the microscopic mechanisms related with deformation-induced phase transformation (i.e., nanocrystallization) have yet to be clearly resolved and understood. Therefore, exploiting the difference of microstructures based on a comparative study between the as-cast state and deformed state of samples subjected to severe deformation under the same preparation condition is helpful to understand the origin of inhomogeneities in the glassy phase. In order to understand the underlying mechanism of phase transformation along the shear bands after deformation, we review on the mechanical strain induced microstructural inhomogeneities, such as structure of shear bands and related nanocrystallization in typical bulk metallic glass and metallic glass matrix composite subjected to room temperature deformation under compressive loading conditions, respectively.

\section{MATERIALS AND METHODS}

MG ribbons were prepared by arc melting buttons of highpurity elements under an Ar atmosphere. Approximately $15 \mathrm{~g}$ of a cast ingot was cut and re-melted in $\mathrm{SiO}_{2}$ (quartz) nozzles to prepare ribbons by single-roller $\mathrm{Cu}$ wheel melt spinning at a linear surface velocity of $40 \mathrm{~ms}^{-1}$. A superheating of approximately $70 \mathrm{~K}$ was used prior to ejecting the liquid onto the rotating $\mathrm{Cu}$ wheel. BMG samples were alloyed by arc melting high-purity elemental constituents under an $\mathrm{Ar}$ atmosphere. These master ingots were re-melted in a $\mathrm{SiO}_{2}$ (quartz) tube by induction heating and the melt was injection cast through a nozzle into a $1 \mathrm{~mm}$ diameter cavity of a $\mathrm{Cu}$ mold. The resulting ingots were $1 \times 45 \mathrm{~mm}$ (diameter $\times$ height). The metallic glass matrix composite (MGMC) samples were produced by hot-extrusion. Powders of the MG alloy were made by high-pressure gas atomization. These powders were sieved to $-63 \mu \mathrm{m}$ and combined with 40 volume $\%$ brass (also $<63 \mu \mathrm{m}$ ) in a turbula blender. The powders were loaded into a copper can and sealed under vacuum for extrusion. Extrusion was performed by heating the billet to a temperature above the glass transition temperature but below the crystallization temperature of the metallic glass.

Test specimens of monolithic Ni-based metallic glass sample were cut and polished to produce $1 \times 2 \mathrm{~mm}$ (diameter $\times$ height) rods for uniaxial compression testing. The MGMC compression test samples have a dimension of $2 \times 2 \times 4 \mathrm{~mm}$. Testing of all samples was performed at a strain rate of $1 \times 10^{-4} \mathrm{~s}^{-1}$. Boron nitride (BN) was used as a lubricant between the sample and the punches. For TEM thin foils of the samples were prepared by ion milling using a Gatan Model 691 PIPS (Gatan, USA) and TEM was performed with Jeol JEM 4010 (JEOL, Japan) and JEM 2000EX (JEOL) microscopes.

\section{RESULTS AND DISCUSSION}

\section{Shear Band Formation in Deformed Metallic Glassy Ribbons and Bulk Metallic Glass}

To understand the formation of shear bands under different stress states, we characterized the shear bands formed in bended Ni-based MG ribbons and in uniaxially compressed Ni-based BMG samples. Fig. 1 displays bright field (BF) TEM images and the corresponding selected area diffraction patterns (SADP) obtained from the bended Ni-based MG ribbon and the uniaxially compressed Ni-based BMG samples. The BF TEM image and the broad halo SADP in Fig. 1A obtained from the compressively deformed region of the 10 times $180^{\circ}$ bend-tested Ni-based MG ribbon shows the typical straight white contrast of shear bands surrounded by undeformed amorphous regions. Even when the strain reaches around $10^{3} \%$ in case of the bending test, no mechanically induced nanocrystals can be observed.

In the case of the uniaxially compressed Ni-based BMG sample, the BF TEM image in Fig. 1B (region A in Fig. 2A), where an ideal uniaxial compressive stress state would be expected, shows primary shear bands that propagated along the maximum shear direction, i.e., under an angle of about $45^{\circ}$, to the loading axis. It is important to note the uniform width (actual thickness of the shear plane) of the shear bands. The inset SADP taken from within the shear band region in Fig. $1 \mathrm{~B}$ suggests that the deformed amorphous material in the shear band is unchanged relative to the adjacent undeformed regions.

Considering the deformation of the BMG specimen under uniaxial compression testing as shown in Fig. 2A, in case of the loading axis (region $\mathrm{C}$ in Fig. 2A), it can be assumed that there is no friction effect between the samples and the punches and the compression will be homogeneous (Steif, 1983). Therefore, this region is similar to isotropic materials where plastic yielding depends only on the magnitudes of the three principal stresses. However, at the lateral surface (regions A, B in Fig. 2A) of the specimen, which is the more 

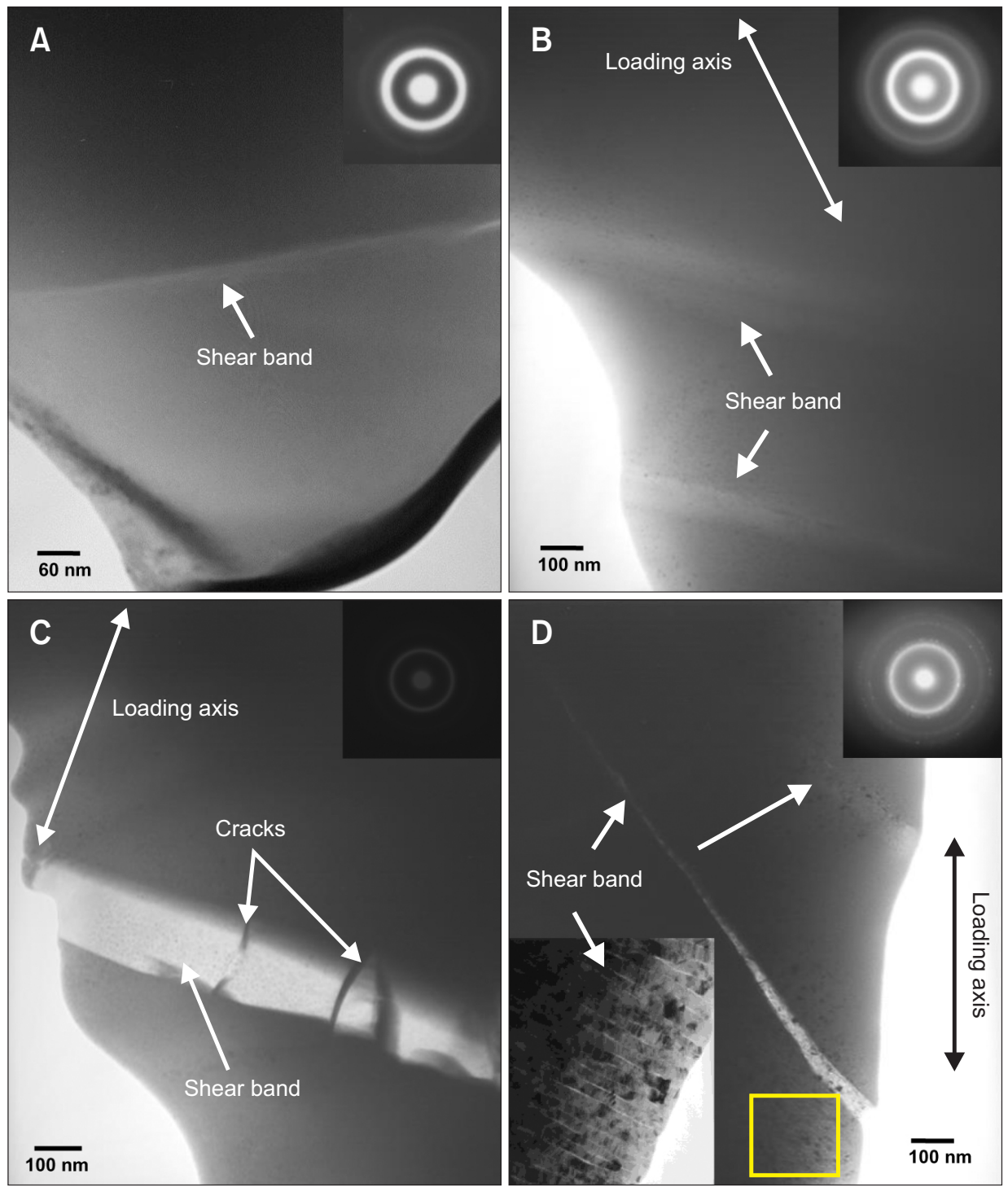

A

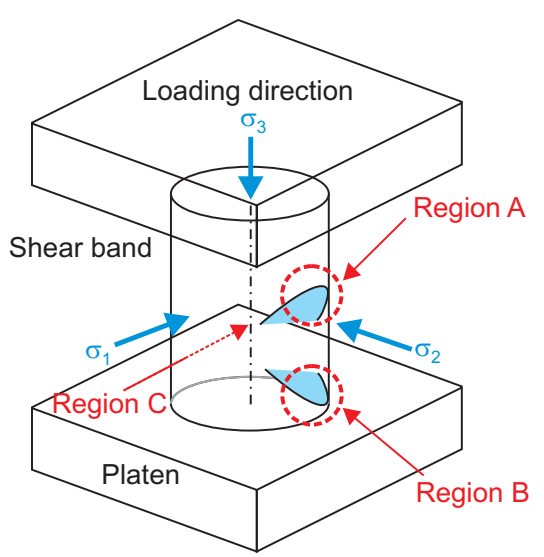

B

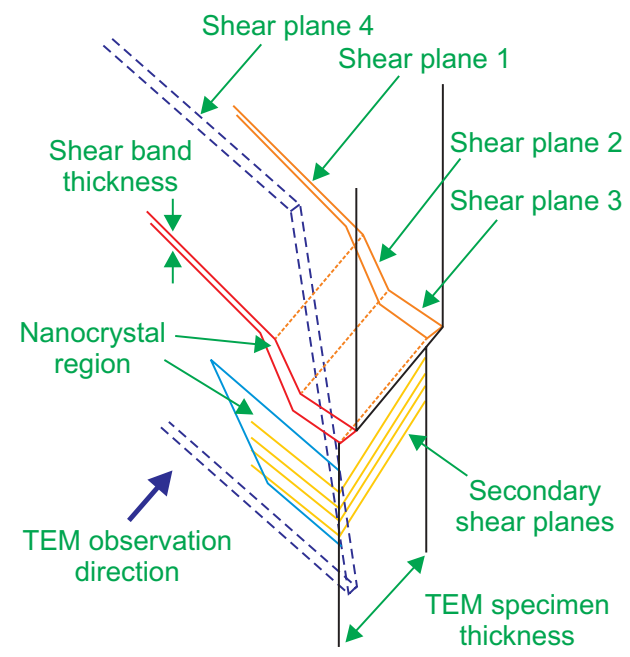

Fig. 1. Bright field (BF) transmission electron microscopy (TEM) images and corresponding selected area diffraction patterns (SADP) obtained from a bended Ni-based metallic glasse (MG) ribbon and an uniaxially compressed Ni-based bulk metallic glass (BMG) sample, respectively (Pauly et al., 2009; Lee et al., 2014). (A) BF TEM image and inset SADP obtained from the Ni-based MG ribbon after deformation. (B) BF TEM image and inset SADP obtained from the Ni-based BMG after deformation illustrating typical shear bands that are without nanocrystals and uniform in width. (C) BMG BF TEM image and corresponding SADP for shear band and fragmentation of a primary shear band obtained in the Ni-based BMG after interrupted the compression test before fracture. (D) BF TEM image and inset SADP obtained from the $\mathrm{Ni}$ based BMG after deformation revealing a shear band that varies in its width and contains nanocrystals; inset image shows enlarged view of yellow square marked area.

Fig. 2. (A) Schematic diagram illustrating for the nominal locations where the two transmission electron microscopy (TEM) samples were obtained (Lee et al., 2014). (B) Schematic diagram of Fig. 1D for the bright field TEM image obtained from the Ni-based bulk metallic glass after deformation revealing a shear band that varies in its width and contains nanocrystals. 
interesting point rather than the loading axis because it was experimentally observed that the shear bands in metallic glass are typically initiated from the surface of the sample (Bao \& Wierzbicki, 2005; Yang et al., 2005), the strains are uniform and the radial stress is zero at that region because of zero surface traction. In the region $\mathrm{A}$, the stress triaxiality reaches a minimum, which corresponds to a pure shear condition. The radial gradient is negligible in the equatorial region due to the rotational symmetry of the specimen, so the stress state reaches essentially plane stress conditions in the equatorial region (Steif, 1983).

\section{Effect of Geometrical Constraints on the Formation of Shear Bands in Bulk Metallic Glass}

When MGs are deformed under a compressive stress state, it is often found that nanocrystallization occurs (Ye \& Lu, 1999; Jiang et al., 2003; Lee et al., 2008). For example, pressure-induced crystallization under hydrostatic pressure has been experimentally found in $\mathrm{Zr}$ - and Al-based MGs (Ye \& Lu, 1999; He et al., 2002) and numerically postulated in simple amorphous systems (Lee et al., 2003; Lee et al., 2004; Boucharat et al., 2005). Also the formation of nanocrystals in bended Al-based amorphous ribbons and beneath indented Zr-based metallic glass alloys has been reported (Kim et al., 2002; Jiang et al., 2003). Such experimental and simulation results indicate that the nucleation of nanocrystals at shear bands during deformation may be determined by geometrical constraint effects resulting from multi-axial stress conditions. Even for simple uniaxial compression testing of BMG samples, the material adjacent to the upper and lower punches is susceptible to non-uniform loading and the effective stress state can deviate significantly from pure uniaxial compressive loading. The evaluation of different regions from the compressed Ni-based BMG illustrates this effect and the corresponding propensity towards nanocrystallization in shear bands that form under multi-axial loading. The friction stress induced by the punches at the lateral surface of the bottom part of the sample during compression testing (region B in Fig. 2A) is defined as $\sigma=\sigma_{a}\left[1+\bar{m} / 3 \sqrt{3} \lambda_{0} \times \exp (3 \bar{\varepsilon} / 2)\right]^{-1}$, where $\sigma$ is the local von Mises equivalent flow stress of the material and $\bar{m}$ the friction parameter $(0 \leq \bar{m} \leq 1), \bar{\varepsilon}$ is effective strain, $\mu$ is the Coulomb friction coefficient $\left(\mu=\bar{m} / 2 \sqrt{1-\bar{m}^{2}}\right)$ at the compressed surface, $\sigma_{a}$ is the apparent flow stress and $\lambda_{0}=H_{0} / D_{0}$ is the initial aspect ratio of the specimen (Chovet et al., 2002; Bao \& Wierzbicki, 2005). In the current study $\sigma_{a}$ is $2 \mathrm{GPa}, \lambda_{0}$ is $2, \bar{\varepsilon}$ is 0.02 for the Ni-BMG and $\mu$ for the used BN lubricant is 0.3 at room temperature. The calculated additional stresses from the friction-corrected effective flow stress for the uniaxial compression of a cylinder at region B is $550 \mathrm{MPa}$. Considering the temperature increase induced by heat generation due to friction, in case of very slow strain rate, it can be assumed that the sliding speed approaches a steady or quasistatic state.
The temperature rise of the contact surface can be calculated by considering the heat generation equation at a single area of contact defined as $T(t)=2 h \sqrt{t} / \sqrt{\pi K \rho C_{p}}$ (Archard, 1959), where $K$ is the thermal conductivity, $\rho$ is the density, $C_{p}$ is the specific heat, and $t$ is the time for which the heat is supplied and can be replaced by $a / v$. At the surface the heat is supplied at a fixed rate with constant heat flux, and the average heat flux $(h)$ at the contact area due to the frictional heating can be expressed as $h=\mu W v / \pi a^{2}$ (Jaeger, 1942), where $\mu$ is the coefficient of friction, $a$ is the contact radius, $v$ is the velocity and $W$ is the load. In the current study $\mu=0.3$ for $\mathrm{BN}, W=1413$ $\mathrm{N}, v=3.3 \times 10^{-4} \mathrm{~mm} / \mathrm{s}, \rho=7.9 \mathrm{~g} / \mathrm{cm}^{3}, a=0.5 \mathrm{~mm}$ for the Ni-based BMG and $K=7 \mathrm{~W} / \mathrm{mK}, C_{p}=500 \mathrm{~J} / \mathrm{kgK}$ for BMGs (Harms et al., 2002). The calculated temperature rise by friction on the contact surface is only $1.48 \mathrm{~K}$, because the accumulation of thermal energy by friction is more dependent on strain rate and contact area rather than the applied stress (Abdel-Aal, 2002). Therefore, the effect of friction induced temperature increases on the crystallization is negligible. As shown in Fig. $1 \mathrm{D}$, the BF TEM image obtained from the lateral surface area (region B in Fig. 2A), which is close to the loading punches, shows the effect of stress triaxiality induced by friction. From the variation of the shear band propagation directions and the sample, additional stress was generated by friction at the punch-specimen interface, which was deformed under the non-uniaxial compressive stress state.

\section{Stress Triaxiality Effect on the Yielding of the Bulk Metallic Glass}

The relationship between the equivalent stress and stress triaxiality is well described by the Mohr-Coulomb criterion, and the stress triaxiality has a linear relation with the hydrostatic pressure (Steif, 1983; Lewandowski \& Lowhaphandu, 2002; Lund \& Schuh, 2003; Lee et al., 2014). The deviation of the shear plane away from the maximum shear direction is an indication that the flow process of the material has a dependency on the normal stress acting on the shear plane (Zhang et al., 2003; Bao \& Wierzbicki, 2005). Moreover, the equivalent strain to failure differs quite significantly for specimens under different stress triaxialities (Bao \& Wierzbicki, 2004). The numerical calculation results revealed that the stress triaxiality differs between the compressive surface and the lateral surface during compression testing (Teng et al., 2005). Therefore, the BF TEM image of the primary shear bands in Fig. 1D obtained from the lateral surface area (region B in Fig. 2A), which is close to the loading punches, reveal that the shear bands propagate with a deviation from the maximum shear direction $\left(\sim 45^{\circ}\right)$ to the loading axis. Moreover, the thickness of these shear bands is not uniform along the propagation direction; they are wider at the base and sharper towards the tip. The inset TEM image in Fig. 1D, which was obtained from 
the deformed region adjacent to the non-uniform primary shear band, reveals finely distributed secondary shear bands populated with nanocrystals.

The shear band morphology in the most deformed MG samples, both in the ribbons and the bulk rods, is similar to that seen in Fig. 1A and B, where the sample is amorphous and the shear bands are straight, with uniform thickness and exhibiting no evidence of crystallization. However, the shear bands shown in Fig. 1D are crooked, taper along their length and contain nanocrystals. It is possible that the propagation shape and uniformity in the thickness of the shear bands indicates the stress state during deformation and geometrical constraints are very likely to be created under multi-axial loading conditions. To understand the complex stress state shown in Fig. 1D, a detailed schematic drawing for the lateral surface area, which is close to the loading punches (region B in Fig. 2A), is shown in Fig. 2B. Here, the shear planes, which correspond to the normal cross-section of shear bands in the BF TEM image (Fig. 1D), deviate from the direction of the maximum shear plane. This suggests that strong stress triaxiality exists in this area because each shear plane developed following its most favorable direction. This reveals that the orientation of the macroscopic shear plane with respect to the loading axis is significantly affected by changes in stress triaxiality.

Fig. 1C shows the BF TEM image for a primary shear band before it developed to the fracture plane obtained from a nearly failed Ni-based BMG (further compressive deformed state from Fig. 1B) after interrupting the compression testing. The inset SADP taken from within the shear band region in Fig. 1C suggests that the amorphous state after deformation is unchanged relative to the adjacent undeformed regions. A shear band shown in Fig. 1C is fractured into several parts by cracks, which run perpendicular to the shear plane. Note that the shear band has a straight-line shape and is uniformly $\sim 150$ $\mathrm{nm}$ thick. However, the apparent thickness of a shear band as visible in TEM can vary due to the alignment of the shear band relative to the observation direction. Nevertheless, the shear band width in Fig. 1C is considerably thicker than most reported 20 to $50 \mathrm{~nm}$ thick shear bands (Pekarskaya et al., 2001) and the width observed in Fig. 1B. In general, the actual failure of a BMG material is a competitive process between shear, distensile and normal tensile fracture depending on the loading mode and inhomogeneity of the microstructure (Zhang et al., 2003). When the critical distensile stress is higher than the critical shear fracture stress, a crack or split is more likely to form in a plane parallel to the loading direction rather than continued shear (Zhang et al., 2003). This fracture model was suggested by Grebe et al. (1985) to result from cracks that are generated parallel to the normal stress direction or perpendicular to the shear stress direction and shear plane in a Ti-based alloy deformed under high stress and strain rate.

When the cracks developed the specimen was quite compressed state by compressive force. However, most of the area of the specimen is still deformed elastically due to the relatively high Poisson's ratio and large elastic limit of the material. When the specimen, not fractured yet but containing several shear bands, is unloaded after interrupted compression for microstructural analysis, most part of the sample volume is elastically unloaded except the shear band region. Due to this, the fragmented pieces within the shear band are superimposed on each other, as shown in Fig. 1C. When the primary shear band transformed into a fracturecausing crack, it releases the maximum strain energy, which is converted to heat energy. Considering the dissipation of heat by conduction, the amount of released energy at the primary shear band is significant. It has been reported that temperature rise in the vicinity of shear bands can induce melting of a Sn coating layer in case of a bend-tested Zr-based BMG and the temperature increase at the primary shear band is sufficient to reach the melting point of the material at the fracture plane (Lewandowski \& Greer, 2006). However, the local heating effect to accompanying phenomena, as a secondary effect, such as the nucleation of nanocrystals sometimes seen after deformation is still unclear (Spaepen, 2006). Therefore as shown in Fig. $1 B$ and C, despite the possible local heating effect, we observed no nucleation of nanocrystals at the shear bands. Because when the shear band conditions approach from uniaxial to constrained multi-axial stress conditions, then strain energy-driven atomic diffusion may indeed dominate the nanocrystallization rather than adiabatic heating induced by shear localization.

\section{Deformation Induced Nanocrystallization in the Bulk Metallic Glass}

During the initial stage of nucleation of crystals in an amorphous phase, crystallization may be governed by (1) the thermodynamic potential barrier of nucleation $\left(\Delta \mathrm{G}^{*}\right)$, (2) the activation energy barrier $\left(Q_{n}\right)$ for atomic diffusion, and (3) the volume change effect related to the interface energy $(\gamma)$ (Ye \& Lu, 1999). For homogeneous nucleation, an applied pressure results in an enhancement of the nucleation rate and in a reduction of the crystallization temperature by the rapid decrease of $\Delta \mathrm{G}^{\star}$ for nucleation thus promoting crystallization. On the other hand, $Q_{n}$ for atomic diffusion increases, thus retarding the crystallization process. So the role of pressure on the nucleation during the crystallization process might be governed by the combination of these two controlling factors. For a primary or eutectic reaction, it is known that $Q_{n}$ for atomic diffusion becomes the dominant factor (Ye \& Lu, 1999). However, its value is small compared to $\Delta \mathrm{G}^{*}$ in case of a polymorphous reaction. Thus, $\Delta \mathrm{G}^{*}$ becomes the rate controlling factor in the entire nucleation 
process. For an extremely small crystalline embryo, the ratio of interface/volume is very high, and the contribution of the interface to the total volume change is significant during the nucleation process. The overall volume change for forming a crystal nucleus from the amorphous phase may be positive when the nucleus size is smaller than a critical size. Such a positive volume change provides an unstable high energy state for the nucleation rate to increase under a higher pressure (Boucharat et al., 2005). To release the very high energy induced by the volume change effect, nano-scale crystals
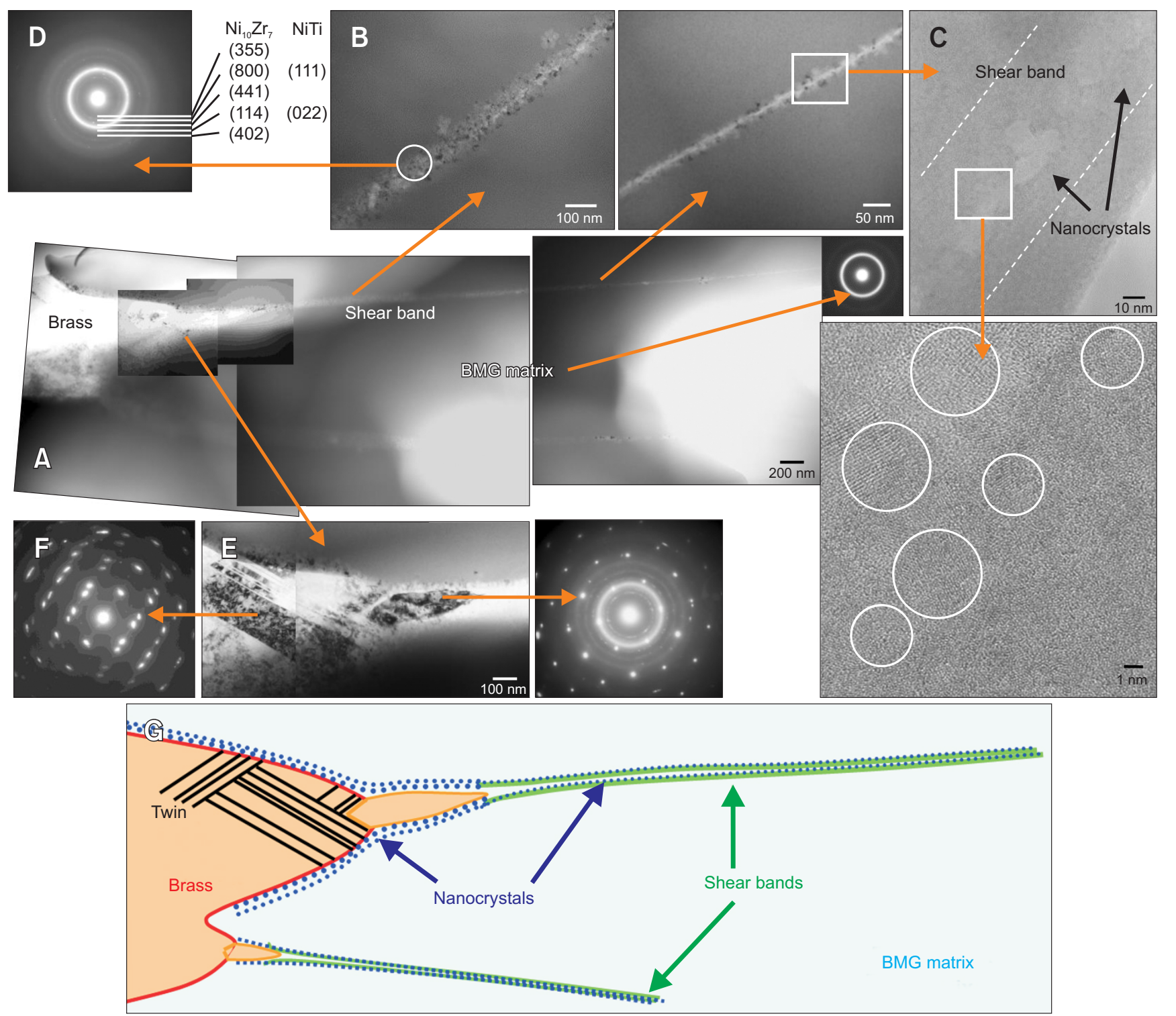

Fig. 3. Bright field transmission electron microscopy (BF TEM) images and inset selected area diffraction patterns (SADPs) obtained from the brass reinforced bulk metallic glass (BMG) composite after deformation; shear bands with nanocrystals, and inhomogeneous width of shear bands, generated at the tip of brass; high-resolution (HR) TEM image of shear band with nanocrystals obtained from the brass reinforced BMG composite. The areas labeled A-E are described in the text (Lee et al., 2008). (A) BF TEM images obtained from the deformed Ni-based metallic glass matrix composite (MGMC) sample showing a shear band that initiated from the sharp edge of a brass particle. (B) BF TEM image obtained from the deformed Ni-based MGMC sample showing the structure and morphology of the nanocrystallites at the shear band. (C) HR TEM image revealing $20 \mathrm{~nm}$ sized nanocrystals that are homogeneously distributed in the amorphous matrix. (D) SADPs taken from the shear band and the surrounding amorphous phase consisting of spotty rings superimposed on the diffuse halo ring from the amorphous phase; the positions of the spotty rings correspond to those of $\mathrm{Ni}(\mathrm{Zr}, \mathrm{Ti})$ and $\mathrm{Ni} \mathrm{i}_{10}\left(\mathrm{Zr}, \mathrm{Ti}_{7}\right.$. $(\mathrm{E})$ BF TEM image obtained from the brass fiber-BMG matrix interface, showing that nanocrystallization of the amorphous phase occurs within $60 \mathrm{~nm}$ of the sharp edge of the brass. (F) The SADP taken from the brass phase marked in Fig. 2E consists of two mirror-related [011] zones indicating the $\{111\}$ twinning of the fcc structure. $(\mathrm{G})$ Schematic diagram illustrating for the brass reinforced BMG composite after deformation at the tip of brass corresponding TEM images of Fig. 3A-F. 
with a lot of interfaces will form. Such an interface plays an important role in the crystallization process, especially for this polymorphous reaction. Eventually nano-scale crystalline phases, which cannot be obtained by conventional annealing (Lee et al., 2004), are observed at the shear band in the current Ni-based BMG. As this Ni-based BMG is known to exhibit a polymorphous crystallization process (Lee et al., 2004), which requires no long range atomic diffusion in contrast to primary or eutectic crystallization, the crystallization of this alloy is basically an interface-controlled transformation (Ye $\& \mathrm{Lu}, 1999)$. This can lead to the nanocrystals observed at the crooked primary shear band in the deformed region in Fig. $1 \mathrm{D}$, similar to the observation of nanocrystals arranged in a "bandlike" structure around the multiple shear bands, as a result of the restraint of shear band propagation owing to the dynamic nanocrystallization in the compressive deformed $\mathrm{Zr}$ based BMG (Saida et al., 2005). The nanocrystals are found several hundred $\mathrm{nm}$ from the shear bands (Fig. 2B) and similar observations of nanocrystals outside shear bands have been reported previously (Boucharat et al., 2005). It has been proposed that these nanocrystals are part of another shear plane that is normal to the TEM viewing direction, which was removed during sample preparation. Finally, only a part of nanocrystallized region remains on the TEM specimen (shown in Fig. 2B).

\section{Nanocrystallization at the Shear Bands in Metallic Glass Matrix Composite}

Further evidence of nanocrystallization under multiaxial stress conditions can be found in the deformed $\mathrm{Ni}$ based MGMC sample. The evolution of pressure beneath the indenter for spherical indenters has shown that nanoindentation clearly produces an order of magnitude larger pressure beneath the spherical indenter (160 GPa) than in the surrounding area (Kramer et al., 2005). The hydrostatic stress level is markedly elevated in the vicinity of a sharp corner of a whisker and can reach up to six times the overall yield strength (Christman et al., 1989). From the microstructure of the Ni-based MGMC material shown in Fig. 3A, the edge corner of a brass fiber surrounded by the matrix can be assumed very similar to the indenter tip of indentation tools and can be expected to induce a high level of hydrostatic pressure induced by multi-axial stresses, even the Ni-based MGMC specimen deformed under quasistatic uniaxial loading condition. A collage of BF TEM images obtained from the deformed Ni-based MGMC sample shows that the shear band initiated from the sharp edge of a brass fiber. Nanometer-size crystalline precipitates are homogeneously distributed within this area; however regions outside the shear band still appear to be completely amorphous (Fig. 3A). Along the brass particle-BMG matrix interface, nanocrystallization of the amorphous phase occurs within $60 \mathrm{~nm}$ of the sharp edge of the brass (Fig. 3B). HRTEM images clearly reveal $\sim 20$ $\mathrm{nm}$-sized nanocrystals that are homogeneously distributed in the amorphous matrix (Fig. 3C). The SADPs taken from the shear band and the surrounding amorphous phase consist of spotty rings superimposed on the diffuse halo ring from the amorphous phase (Fig. 3D). The positions of the spotty rings correspond to those of $\mathrm{Ni}(\mathrm{Zr}, \mathrm{Ti})$ and $\mathrm{Ni}_{10}(\mathrm{Zr}, \mathrm{Ti})_{7}$ compounds. The structure and morphology of the nanocrystallites inside the shear band are the same as those previously observed after isothermal annealing of an amorphous $\mathrm{Ni}_{59} \mathrm{Zr}_{20} \mathrm{Ti}_{16} \mathrm{Si}_{2} \mathrm{Sn}_{3}$ sample (Lee et al., 2004). As shown in Fig. 3, the thickness of the primary shear band is larger at the sharp tip of the brass than inside the matrix, farther away from the brass (Fig. 3E). The SADP taken from the brass phase marked in Fig. 3E consists of two mirror-related [011] zones indicating $\{111\}$ twinning of the fcc structure (Fig. 3F). Within the primary shear band the volume fraction of nanocrystals is much higher near the brass particle than further into the matrix. These nanocrystals and the shear band initiation at the brass particles indicate that upon yielding highly confined strain energy saturates and the local stress reaches a very high level.

\section{CONCLUSIONS}

In the current study, we examined the evolution and structure of shear bands and related nanocrystallization in Ni-based MG ribbons, in $\mathrm{Ni}_{59} \mathrm{Zr}_{20} \mathrm{Ti}_{6} \mathrm{Si}_{2} \mathrm{Sn}_{3}$ BMG and for a ductile phase-reinforced Ni-based metallic glass matrix composite (MGMC; $\mathrm{Ni}_{59} \mathrm{Zr}_{20} \mathrm{Ti}_{16} \mathrm{Si}_{2} \mathrm{Sn}_{3}$ reinforced with 40 vol.\% brass) deformed under compressive loading conditions, respectively. Experimental results on the deformation of metallic glass suggest that the occurrence of nanocrystallization at a shear band implies the loading condition that induces deformation is more triaxial in nature than uniaxial. Therefore, under a compressive stress state, geometrically constrained strain induced atomic diffusion generated by the stress triaxiality plays an important role in the deformation-induced nanocrystallization at the shear bands. The present results support the significant role of localized strain that increases by deformation under multi-axial stress conditions, which promotes nanocrystallization.

\section{CONFLICT OF INTEREST}

No potential conflict of interest relevant to this article was reported. 


\section{REFERENCES}

Abdel-Aal H A (2002) Thermal kinetics of protective oxide layer formation in the dry sliding of metallic tribo-specimens. Tribol. Int. 35, 757-769.

Agron A S (1979) Plastic deformation in metallic glasses. Acta Metall. 27, 47-58.

Archard J F (1959) The temperature of rubbing surfaces. Wear 2, 438-455.

Bao Y and Wierzbicki T (2005) On the cut-off value of negative triaxiality for fracture. Eng. Fract. Mech. 72, 1049-1069.

Bao $Y$ and Wierzbicki $Y$ (2004) On fracture locus in the equivalent strain and stress triaxiality space. Int. J. Mech. Sci. 46, 81-98.

Boucharat N, Hebert R, Rösner H, Valiev R, and Wilde G (2005) Nanocrystallization of amorphous Al88Y7Fe5 alloy induced by plastic deformation. Scr. Mater. 53, 823-828.

Chen H, He Y, Shiflet G J, and Poon S J (1994) Deformation-induced nanocrystal formation in shear bands of amorphous alloys. Nature 367, 541-543.

Chovet C, Desrayaud Ch, and Montheillet F (2002) A mechanical analysis of the plane strain channel-die compression test: friction effects in hot metal testing. Int. J. Mech. Sci. 44, 343-357.

Christman T, Needleman A, and Suresh S (1989) An experimental and numerical study of deformation in metal-ceramic composites. Acta Metall. 37, 3029-3050.

Conner R D, Johnson W L, Paton N E, and Nix W D (2003) Shear bands and cracking of metallic glass plates in bending. J. Appl. Phys. 94, 904-911.

Deng D, Argon A S, and Yip S (1989) Simulation of plastic deformation in a two-dimensional atomic glass by molecular dynamics IV. Phil. Trans. R. Soc. Lond. A 329, 613-640.

Donovan P E (1989) A yield criterion for Pd4ONi4OP20 metallic glass. Acta Metall. 37, 445-456.

Grebe H A, Pak H R, and Meyers M A (1985) Adiabatic shear localization in titanium and Ti-6 pct Al-4 pct V alloy. Matall. Trans. A 16, 761-775.

Greer A L (1995) Metallic glasses. Science 267, 1947-1953.

Harms U, Shen T D, and Schwarz R B (2002) Thermal conductivity of Pd4ONi40-xCuxP2O metallic glasses. Scr. Mater. 47, 411-414.

He D, Zhao Q, Wang W H, Che R Z, Liu J, Luo X J, and Wang W K (2002) Pressure-induced crystallization in a bulk amorphous Zr-based alloy. J. Non-Cryst. Solids 297, 84-90.

Jaeger J C (1942) Moving sources of heat and the temperature at sliding contacts. Proc. R. Soc. NSW. 76, 203-224.

Jiang W H, Pinkerton F E, and Atzmon M (2003) Effect of strain rate on the formation of nanocrystallites in an Al-based amorphous alloy during nanoindentation. J. Appl. Phys. 93, 9287-9290.

Kim J -J, Choi Y, Suresh S, and Argon A S (2002) Nanocrystallization during nanoindentation of a bulk amorphous metal alloy at room temperature. Science 295, 654-657.

Kramer M J, Sordelet D J, Bastarows A F, Tan X, and Biner S B (2005) Absence of crystallization during cylindrical indentation of a Zr-based metallic glass. J. Non-Cryst. Solids 351, 2159-2165.

Lee B J, Lee C S, and Lee J C (2003) Stress induced crystallization of amorphous materials and mechanical properties of nanocrystalline materials: a molecular dynamics simulation study. Acta Mater. 51, 6233-6240.

Lee B J, Lee J C, Kim Y C, and Lee S H (2004) Behavior of amorphous materials under hydrostatic pressures: a molecular dynamics simulation study. Met. Mater. Int. 10, 467-474.

Lee J K, Bae D H, Yi S, Kim W T, and Kim D H (2004) Effects of Sn addition on the glass forming ability and crystallization behavior in $\mathrm{Ni}-\mathrm{Zr}$-Ti-Si alloys. J. Non-Cryst. Solids 333, 212-220.

Lee M H, Bae D H, Kim D H, Kim W T, Sordelet D J, Kim K B, and Eckert $J$ (2008) Nanocrystallization at shear bands in bulk metallic glass matrix composites. Scripta Mater. 58, 651-654.

Lee M H, Kim B S, Kim D H, Ott R T, Sansoz F, and Eckert J (2014) Effect of geometrical constraint condition on the formation of nanoscale twins in the Ni-based metallic glass composite. Philos. Mag. Lett. 94, 351-360.

Lee M H, Lee J K, Kim K T, Thomas J, Das J, Kühn U, and Eckert J (2009) Deformation-induced microstructural heterogeneity in monolithic Zr44Ti11Cu9.8Ni10.2Be25 bulk metallic glass, Phys. Status Solidi $R R L$ 3, 46-48.

Lewandowski J J and Greer A L (2006) Temperature rise at shear bands in metallic glasses. Nat. Mater. 5, 15-18.

Lewandowski J J and Lowhaphandu P (2002) Effects of hydrostatic pressure on the flow and fracture of a bulk amorphous metal. Philos. Mag. A 82, 3427-3441.

Liu C T, Heatherly L, Horton J A, Easton D S, Carmichael C A, Wright J L, Schneibel J L, Yoo M H, Chen C H, and Inoue A (1998) Test environments and mechanical properties of Zr-base bulk amorphous alloys. Metall. Mater. Trans. A 29, 1811-1820.

Lund A C and Schuh C A (2003) Yield surface of a simulated metallic glass. Acta Mater. 51, 5399-5411.

Pauly S, Lee M H, Kim D H, Kim K B, Sordelet D J, and Eckert J (2009) Crack evolution in bulk metallic glasses. J. Appl. Phys. 106, 103518.

Pekarskaya E, Kim C P, and Johnson W L (2001) In situ transmission electron microscopy studies of shear bands in a bulk metallic glass based composite. J. Mater. Res. 16, 2513-2518.

Saida J, Setyawan A D H, Kato H, and Inoue A (2005) Nanoscale multistep shear band formation by deformation-induced nanocrystallization in Zr-Al-Ni-Pd bulk metallic glass. Appl. Phys. Lett. 87, 151907.

Spaepen F (1977) A microscopic mechanism for steady state inhomogeneous flow in metallic glasses. Acta Metall. 25, 407-415.

Spaepen F (2006) Metallic glasses: Must shear bands be hot. Nat. Mater. 5, 7-8.

Steif P S (1983) Ductile versus brittle behavior of amorphous metals. J. Mech. Phys. Solids 31, 359-388.

Teng X, Weirzbicki T, Hiermaier S, and Rohr I (2005) Numerical prediction of fracture in the Taylor test. Int. J. Solids Struct. 42, 2929-2948.

Vaidyanathan R, Dao M, Ravichandran G, and Suresh S (2001) Study of mechanical deformation in bulk metallic glass through instrumented indentation. Acta Mater. 49, 3781-3789.

Yang B, Morrison M L, Liaw P K, Buchanan R A, Wang G, Liu C T, and Denda M (2005) Dynamic evolution of nanoscale shear bands in a bulk-metallic glass. Appl. Phys. Lett. 86, 141904.

Ye F and Lu K (1999) Pressure effect on crystallization kinetics of an AlLa-Ni amorphous alloy. Acta Mater. 47, 2449-2454.

Zhang Z F, He G, Eckert J, and Schultz L (2003) Fracture mechanisms in bulk metallic glassy materials. Phys. Rev. Lett. 91, 045505. 